\title{
Development and Optimisation of Spironolactone Nanoparticles for Enhanced Dissolution Rates and Stability
}

\author{
H. R. Kelidari, ${ }^{1}$ M. Saeedi, ${ }^{2}$ J. Akbari, ${ }^{2}$ K. Morteza-semnani, ${ }^{2}$ H. Valizadeh, ${ }^{3}$ Mohammed Maniruzzaman, ${ }^{4}$ \\ Ali Farmoudeh, ${ }^{2}$ and Ali Nokhodchi, ${ }^{4,5,6}$
}

Received 6 June 2016; accepted 17 August 2016; published online 10 November 2016

\begin{abstract}
Stable solid lipid nanoparticles (SLNs) and nanostructured lipid carriers (NLCs) formulations to enhance the dissolution rates of poorly soluble drug spironolactone (SP) were being developed. Probe ultra-sonication method was used to prepare SLNs and NLCs. All NLCs contained stearic acid (solid lipid carrier) and oleic acid (liquid lipid content), whereas, SLNs were prepared and optimised by using the solid lipid only. The particles were characterised in terms of particle size analysis, thermal behaviour, morphology, stability and in vitro release. The zeta sizer data revealed that the increase in the concentration of oleic acid in the formulations reduced the mean particle size and the zeta potential. The increase in concentration of oleic acid from 0 to $30 \%(w / w)$ resulted in a higher entrapment efficiency. All nanoparticles were almost spherically shaped with an average particle size of about $\sim 170 \mathrm{~nm}$. The DSC traces revealed that the presence of oleic acid in the NLC formulations resulted in a shift in the melting endotherms to a higher temperature. This could be attributed to a good long-term stability of the nanoparticles. The stability results showed that the particle size remained smaller in NLC compared to that of SLN formulations after 6 months at various temperatures. The dissolution study showed about a 5.1- to 7.2-fold increase in the release of the drug in $2 \mathrm{~h}$ compared to the raw drug. Comparing all nanoparticle formulations indicated that the NLC composition with a ratio of 70:30 (solid:liquid lipid) is the most suitable formulation with desired drug dissolution rates, entrapment efficiency and physical stability.
\end{abstract}

KEY WORDS: drug release; solid lipid nanoparticles; spironolactone; stability.

\section{INTRODUCTION}

With the recent advent of high-throughput computational chemistry, the numbers of the poorly water-soluble or insoluble lead compounds have significantly risen. Spironolactone (SP) falls into this group due to its very low aqueous solubility and slow dissolution rate resulting in a

${ }^{1}$ Pharmaceutical Sciences Research Centre, Mazandaran University of Medical Sciences, Sari, Iran.

${ }^{2}$ Faculty of Pharmacy, Mazandaran University of Medical Sciences, Sari, Iran.

${ }^{3}$ Drug applied Research Centre and Faculty of Pharmacy, Tabriz University of Medical Sciences, Tabriz, Iran.

${ }^{4}$ Pharmaceutics Research Laboratory, Arundel Building, School of Life Sciences, University of Sussex, Brighton, BN1 9QG, UK.

${ }^{5}$ Drug Applied Research Center and Faculty of Pharmacy, Tabriz University of Medical Sciences, Tabriz, Iran.

${ }^{6}$ To whom correspondence should be addressed. (e-mail: a.nokhodchi@sussex.ac.uk) variable and poor oral bioavailability (1-6). SP has widely been used over the last two decades to primarily treat edema, hypertension and cutaneous disorders (e.g. acne, hirsutism and androgenic alopecia) in the form of a tablet. Recent studies revealed that about $40 \%$ of the drug candidates are failing out the discovery pipeline due to their limited aqueous solubility. Thus, the solubility enhancement of poorly watersoluble SP is one of the key challenges in pharmaceutical research (7). Various techniques have been reported to increase the dissolution rate of poorly water-soluble SP, such as cyclodextrin complexation approach, freeze-drying or lyophilisation, solid dispersion strategy, liposomal formulations and solid lipid nanoparticles (SLNs) approach $(2-4,8)$. Bourezg et al. prepared a SP-loaded SLN formulation and reported as one of the efficient ways to enhance the dissolution of water-insoluble drugs (6). Moreover, lipid nanoparticles (SLN and NLC) have the capability to enhance the dissolution rate by overcoming the low and variable oral bioavailability of various poorly water-soluble drugs $(4,9)$. The increase in the dissolution is mainly due to a significant 
reduction in the drug particle size down to the nanorange leading to the higher surface area available for dissolution. In general, SLNs are produced using lipid carrier matrices that are solid at both body and room temperatures. An optimised combination of a solid lipid with a liquid lipid forms nanoparticles with more internal defects known as NLC (10). These lipid nanocarriers are produced by partially interchanging the solid and liquid phases. One of the major limitations of SLN approach is the low drug loading capacity. Also, the drug leaking upon cooling and the possible negative effects of polymorphic transformations of the lipid used in the SLN are considered some drawbacks of the traditional SLN approach (11). NLC can be used as an alternative efficient technique that contains the complex lipids and has a high drug loading capacity compared to that of SLNs (11). In addition to the high drug loads, this NLC can contain up to $95 \%$ solid $(12,13)$.

Generally, most of NLC and SLN formulations are not stable at high temperature or on storage. This instability can modify the dissolution behaviour of particles due to aggregation leading to a poor dissolution. Therefore, in the present work, attempts have been made to prepare the SP-loaded SLNs and NLCs by means of a probe-ultrasonication method to enhance the dissolution and stability of these nanoformulations. For the purpose of this study, stearic acid was used as a solid lipid while oleic acid as liquid lipid. To this end, effects of oleic acid concentration on the particle size were investigated and the physico-chemical properties of the bulk SP and SP-loaded lipid nanoparticles were studied. To explore more advantages for nanoformulations, their physical stability during 6 months of storage at various temperatures was also investigated.

\section{MATERIALS AND METHODS}

\section{Materials}

SP was generously provided by Behdashtkar Co. (Pasdaran, Tehran, Iran). Compritol ${ }^{\circledR} 888$ ATO, Lipocire and Precirol ${ }^{\circledR}$ ATO 5 were supplied from Gattefossé (SaintPriest, Cedex, France). Stearic acid, oleic acid, Tween 80 (Tn80) and Span 80 (Sn80) were bought from Merck Co. (Germany). HPLC-grade acetonitrile was supplied from Merck Company. A Milli-Q system (Millipore, Direct-Q) was used to purify the distilled water. All solvents were either of analytical or high-performance liquid chromatography (HPLC) grades and were used as received.

\section{Lipid Screening}

The solubility of SP was studied in different solid lipid matrices with different proportion of oleic acid (0:100; 10:90; $20: 80 ; 30: 70)$ to select the most suitable lipid with a high encapsulation efficiency. Four different solid lipid matrices, namely Compritol ${ }^{\circledR} 888$ ATO, Lipocire, Precirol ${ }^{\circledR}$ ATO 5 and stearic acid were used to determine the saturation solubility of SP keeping the drug concentration constant at $25 \% \mathrm{w} / \mathrm{w}$ (only in this concentration, the saturated solution of the drug can be obtained in the presence of stearic acid). For solid lipid screening tests, certain amounts of SP were added to the lipids and the mixtures were melted at $85^{\circ} \mathrm{C}$ under stirring condition. The presence of drug crystals was visually investigated to see which lipid is able to dissolve the added drug crystals completely. Furthermore, the solubility of SP in the molten solid lipid in combination with oleic acid as liquid lipid (90:10, 80:20 and 70:30) was assessed. The lipid mixtures were stirred using hot plate magnetic stirrer (Unimax 1010, Heidolph, Germany) at $85^{\circ} \mathrm{C}$ and $200 \mathrm{rpm}$. The presence of drug crystals in the molten lipid was visually assessed (see Table I).

\section{Preparation of SLN and NLC}

Spironolactone-loaded SLNs (SP-SLN) and NLCs (SPNLCs) were manufactured and optimised via a process utilising a probe ultrasonication. The method has been adopted from the previously reported studies in literature (6,14-16). To prepare SLNs and NLCs, a carrier lipid in its solid form was melted at $85^{\circ} \mathrm{C}$ either alone or in combination with liquid lipid (oleic acid) and Span 80. The drug was then added and stirred until a complete dissolution was achieved. The aqueous solution of $\operatorname{Tn} 80$ (Tween 80 ) was then added to lipid phase under vigorous stirring using a high shear stress homogeniser Ultra-Turrax ${ }^{\circledR}$ (IKA, Heidelberg, Germany) for $5 \mathrm{~min}$ at $10,000 \mathrm{rpm}$. Upon optimising the homogenous mixture, the heated solution was then sonicated (Bandelinsonopuls, Berlin, Germany) for $5 \mathrm{~min}$ prior to dispersing it into an ice-cold surfactant solution ( $\operatorname{Tn} 80)$. When the solution was cooled to ambient temperature, a further sonication for $10 \mathrm{~min}$ was applied to yield the lipid nanoparticles. For more detail refer to Table II.

\section{Particle Size and Zeta Potentials}

A Zeta Sizer Nano ZS (Malvern Instruments, UK) was used to measure the average particle size and zeta potential of all SLNs and NLCs formulations at room temperature. All samples were run in triplicate and the results shown in Table II are the mean and SD values.

\section{High-Performance Liquid Chromatography Analysis of SP}

The drug content dissolved in the lipid nanoparticles were determined by using a HPLC assay. The HPLC was performed with an Agilent 1100 (Knauer, Berlin, Germany) equipped with an XDB-C18 column from Agilent $(5 \mu \mathrm{m}$,

Table I. Solid Lipid Screening for the Solubility Assessment of SP

\begin{tabular}{lllll}
\hline Lipid $25 \mathrm{mg}$ SP/100 mg solid:liquid lipid & & \\
\hline & $100: 00$ & $90: 10$ & $80: 20$ & $70: 30$ \\
\hline Compritol $^{\circledR}$ 888 ATO & - & - & + & + \\
Lipocire & - & - & - & - \\
Precirol $^{\circledR}$ ATO 5 & - & - & - & - \\
Stearic acid & + & + & + & + \\
\hline
\end{tabular}

$(+)$ indicates the drug is soluble in the phase, $(-)$ indicates the drug is not soluble in the phase

$S P$ spironolactone 
Table II. Formulation Composition and the Physico-chemical Characterization Data of the Prepared SP-Loaded SLN and NLC (\% $w / w$ )

\begin{tabular}{lllllllllr}
\hline Formula & SP & SA & OA & Sn80 & Tn80 & PS $(\mathrm{nm})$ & PI $(\mathrm{nm})$ & ZP $(\mathrm{mV})$ & EE $(\%)$ \\
\hline SP-SLN & 0.4 & 6.0 & - & 2.5 & 5 & $311.8 \pm 19.0$ & $0.199 \pm 0.030$ & $-13.6 \pm 2.6$ & $77.1 \pm 2.6$ \\
SP-NLC1 & 0.4 & 5.4 & 0.6 & 2.5 & 5 & $288.0 \pm 19.9$ & $0.260 \pm 0.050$ & $-17.3 \pm 1.8$ & $84.7 \pm 6.7$ \\
SP-NLC2 & 0.4 & 4.8 & 1.2 & 2.5 & 5 & $240.2 \pm 49.3$ & $0.203 \pm 0.011$ & $-22.1 \pm 4.3$ & $88.7 \pm 4.2$ \\
SP-NLC3 & 0.4 & 4.2 & 1.8 & 2.5 & 5 & $146.4 \pm 15.2$ & $0.225 \pm 0.012$ & $-35.1 \pm 3.0$ & $90.6 \pm 3.5$ \\
\hline
\end{tabular}

$S P$ spironolactone, $S A$ stearic acid, $O A$ oleic acid, $S n 80$ Span $80, T n 80$ Tween $80, P S$ particle size, $P I$ polydispersity index, $Z P$ zeta potential, $E E$ entrapment efficiency, $S L N$ solid lipid nanoparticle, $N L C$ nanostructured lipid carrier

$4.6 \mathrm{~mm} \times 250 \mathrm{~mm})$. The mobile phase used was acetonitrile:millipore water 70:30 $(v / v)$. The UV wavelength was set at $238 \mathrm{~nm}$ to detect the drug with a flow rate of $0.8 \mathrm{ml} /$ $\mathrm{min}$ and the retention time of $7 \mathrm{~min}$.

\section{Determination of SP Entrapment Efficiency}

To determine the SP entrapment efficiency (EE) in all SLNs and NLCs prepared, the amount of free unloaded drug was assessed by a centrifugation process. For this, SP-SLNs and SP-NLCs were centrifuged by using a HERMLE, Z36HK machine (Germany) for $20 \mathrm{~min}$ at $25,000 \mathrm{rpm}$, followed by a filtration with pore size $0.22 \mu \mathrm{m}(n=3)$. The drug content in the supernatant was determined by HPLC method as described above.

The EE of nanoparticles was calculated from Eq. (1).

$\mathrm{EE}=\frac{\mathrm{Wi}-\mathrm{Wf}}{\mathrm{Wi}} \times 100$

Where, Wi and Wf are the initial drug amount and the drug present in supernatant, respectively.

\section{Morphology Measurement}

A field emission scanning electron microscope (FESEM, HITACHI S-4160) was used to analyse the surface of the prepared nanoparticles and the particle morphology. In order to prepare the samples for analysis, a drop of suspension of all formulations was placed on a double-sided carbon tape followed by an overnight drying step at $25^{\circ} \mathrm{C}$. Prior to conducting the SEM study, the particles were subjected to a sputter coating with gold for $40 \mathrm{~s}$. The images were taken at $30 \mathrm{kV}$ at a magnification of $\times 30,000$.

\section{DSC Measurement}

The solid state of the raw drug, lipids, lyophilised SPSLNs and SP-NLCs were studied by a differential scanning calorimetry (DSC 204 F1, Netzsch, Germany). Approximately, 3-12 mg of solid samples were accurately weighed and placed into a standard aluminium pan and sealed. All thermal transitions were recorded at a heating rate of $10^{\circ} \mathrm{C} / \mathrm{min}$ and all samples were heated from 30 and $220^{\circ} \mathrm{C}$ under $\mathrm{N}_{2}$ atmosphere.

\section{Stability Study of SLN and NLC}

The physical stability of SP-loaded lipid nanoparticles were studied at various temperatures $\left(4,25\right.$ and $\left.40^{\circ} \mathrm{C}\right)$ for 6 months. Approximately $5 \mathrm{ml}$ of nanoparticle suspension were filled into glass vials and stored for 6 months. The concentration of the nanoparticles was adjusted at $2 \mathrm{mg} / \mathrm{ml}$. At the end of the stability study period, the particle size analysis was conducted to monitor the effect of the storage condition on the solid lipid nanoparticles.

\section{In Vitro Release Studies}

In vitro dissolution studies were conducted by following the protocol of the USP II (DH1520 dissolution apparatus, ERWEKA, Germany). Lyophilized SP-SLNs and SP-NLCs equivalent to $10 \mathrm{mg}$ of $\mathrm{SP}$ were put into the vessels containing $900 \mathrm{ml}$ of $0.1 \mathrm{M} \mathrm{HCl}$ solution at $37.0 \pm 0.2^{\circ} \mathrm{C}$ with a paddle rotation speed of $100 \mathrm{rpm}$. At different time intervals (20, 40, $60,80,120,240$ and $1440 \mathrm{~min})$, the samples $(1.5 \mathrm{ml})$ were withdrawn from the dissolution vessels, centrifuged (20 min at $25,000 \mathrm{rpm}$ ) and filtered (pore size $0.22 \mu \mathrm{m}$ ). The concentration of SP was determined using a HPLC method as described above at $238 \mathrm{~nm}$.

\section{Statistical Analysis}

The treated groups were compared with the reference sample (control) by an analysis of variance (ANOVA), following Dunnet's test. The SPSS software was used to perform all statistical analyses. The $P$ value $<0.05$ was considered as significant. All the results are expressed as mean \pm standard deviation.

\section{RESULTS AND DISCUSSION}

A low drug payload, drug leakage on cooling etc. are considered as some of the major challenges in the development of SLNs. So, nowadays, an alternative to SLNs, an NLC drug delivery system based on liquid lipid-solid lipid mixture may solve the limitations associated with the conventional SLNs. Unlike SLN, NLC contains the complex lipids. With the presence of oil in these mixtures a melting point depression compared to the pure solid lipid is observed in NLC without compromising the solid state of the final lipid compositions $(11,12)$. Typically, at low concentrations of oil present in the NLC formulations, the oil molecules are distributed within the solid lipid matrix. An adverse effect can be observed when increasing the oil concentration due to 


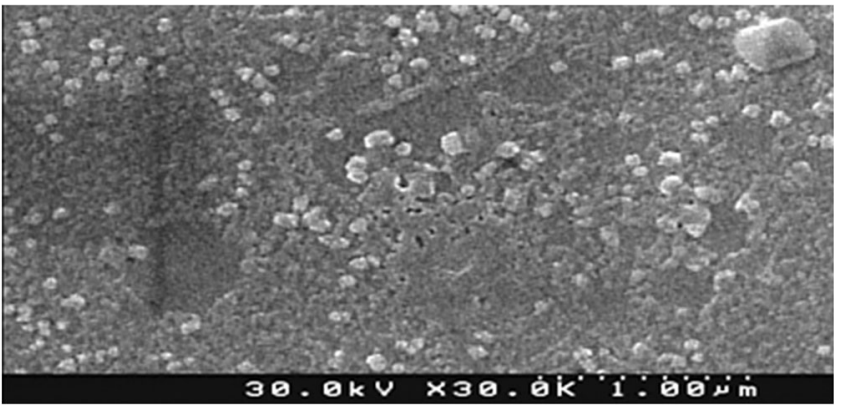

Fig. 1. FESEM images of SP-NLC3 particles under high magnification

the phase separation resulting in the formation of an oily nanocompartments are formed (13).

Therefore, the assessment of the most suitable lipid carriers to prepare stable nanoparticles was optimised as the first step. As shown in Table I, various lipids with different physical and chemical properties were selected. Unlike other lipids, stearic acid performed better in terms of producing a homogenous suspensions showing no drug crystals upon heating with the drug. Therefore, stearic acid was selected as the most suitable solid lipid to prepare SLNs. All other lipids as shown in Table I failed to dissolve SP completely.

It could be clearly observed from Table II that the average particle size and zeta potential decreased with an increase in the concentration of oleic acid $(P>0.05)$. These results were in agreement with previously reported studies (17). An increase in the liquid phase (oleic acid) in the formulations reduced the viscosity of the NLC suspensions and thus the surface tension and the particle size with smoother surfaces (17). However, Yuan et al. indicated that no obvious change in particle size was found when the oleic acid content in NLC was further increased (18). In a separate study, it has been reported that when oleic acid at concentrations of 25 and $50 \%(w / w)$ was used no significant changes were observed on the average diameter and polydispersity index of NLC (19). The phenomenon for zeta potential can be explained by this fact that oleic acid has negative charge because of its carboxylic groups. NLC3 revealed the highest zeta potential values, possibly due to the accumulation of oleic acid at the surface of the nanoparticles (19). It is evident that the increased concentration of oleic acid resulted in the increased drug entrapment efficiency (Table II) $(P>0.05)$. It has been confirmed that the presence of liquid phase in the formulation provides enough space to accommodate the drug particles in the porous network of the system leading to an improved drug entrapment efficiency (17). The solubility of the drug in the liquid lipid could probably be considered as another key factor for the improved entrapment efficiency.

Particle morphology of the optimal formulation (SPNLC3) is shown in Fig. 1. The average particle size is about $150 \mathrm{~nm}$ (particle size range $110-175 \mathrm{~nm}$ ) with almost spherical shape and narrow size distribution. The SEM image showed that some of the nanoparticles were aggregated which could be due to the lipid nature of the carriers used in the presence study. This aggregation could also be due to the sample preparation prior to SEM analysis. Some of the particles in Fig. 1 are departed from being complete spherical shape which has also been reported for NLC (20).

Figure 2 shows the DSC thermal transitions of the raw drug (SP), stearic acid, SP-SLN and the optimised SP-NLC3 formulations. The endothermic event shown at around $213.2^{\circ} \mathrm{C}$ corresponds to the melting point of pure spironolactone (refer to Table III for more detail). In case of SP-SLN and SP-NLC3, no endothermic peak was observed in the melting range of SP (Fig. 3 and Table III). This could be attributed to the entrapment of the drug within the lipid. It could also be claimed that the drug may have been in the amorphous form homogeneously dispersed in lipid matrices (21). Pure stearic acid showed a sharp endothermic event around $75^{\circ} \mathrm{C}$ corresponding to its melting endotherm. The corresponding melting endotherms of each crystalline components in the formulations shifted slightly at a lower temperature (Table III). These differences are generally ascribed to the nanometric size of the particles and also partially could be due to the percentage of oleic acid in the formulation. This is in a good agreement with published data indicating a reduction in stearic acid melting point could be related to the content of oleic acid in the formulations (17).

Figure 3 shows the particle size of two formulations (SPSLN and SP-NLC3) against storage time. The figure shows that the storage temperature and the storage time played a big role in the size growth of nanoparticles. For example, when the storage time was increased from 3 to 6 months at $40^{\circ} \mathrm{C}$, the size of SP-SLNs significantly increased from around 400 to around $900 \mathrm{~nm}(P<0.05)$. Similar trend was observed

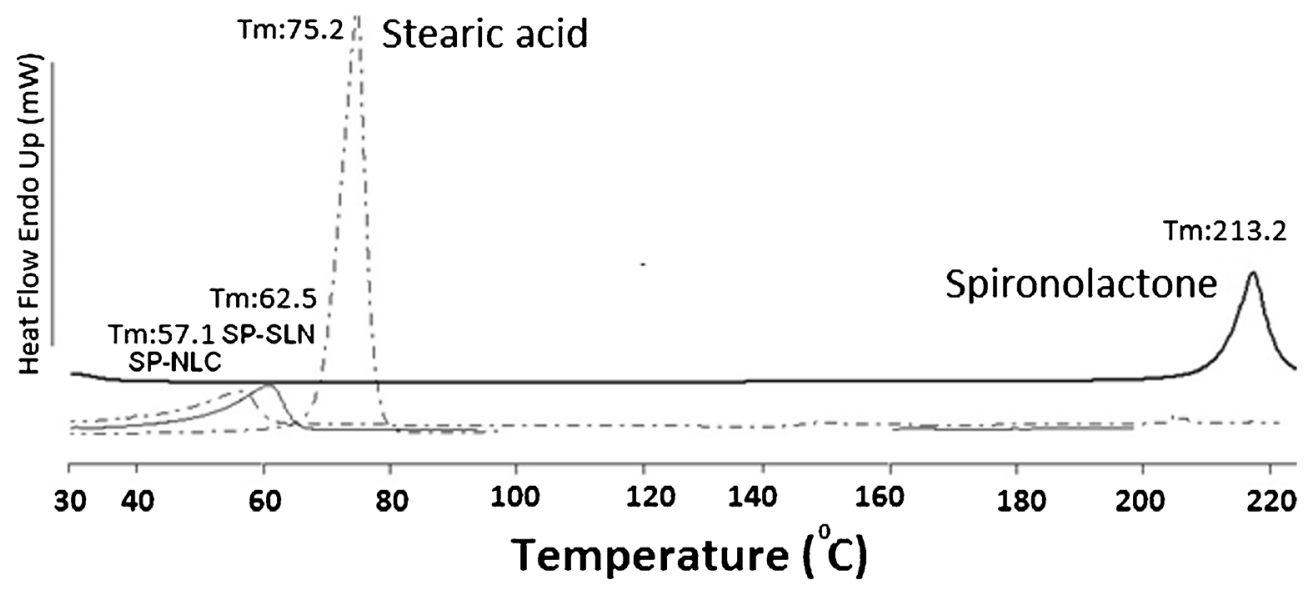

Fig. 2. DSC data of the stearic acid, SP and SP-loaded SLN and NLC3 
Table III. DSC Thermal Events of the Bulk Materials and the Formulations

\begin{tabular}{lllll}
\hline Formula & OA $(\%)$ & Onset $\left({ }^{\circ} \mathrm{C}\right)$ & $\begin{array}{l}\text { Melting } \\
\text { point }\left({ }^{\circ} \mathrm{C}\right)\end{array}$ & $\begin{array}{l}\text { Enthalpy } \\
(\mathrm{J} / \mathrm{g})\end{array}$ \\
\hline Spironolactone & - & 209.44 & 213.24 & 386.77 \\
Stearic acid & - & 69.90 & 75.25 & 255.71 \\
SP-SLN & 0 & 52.97 & 62.55 & 76.11 \\
SP-NLC1 & 10 & 50.94 & 61.59 & 60.02 \\
SP-NLC2 & 20 & 48.95 & 59.23 & 52.86 \\
SP-NLC3 & 30 & 44.76 & 57.10 & 46.33 \\
\hline
\end{tabular}

$S P$ spironolactone, $O A$ oleic acid, $S L N$ solid lipid nanoparticle, $N L C$ nanostructured lipid carrier

for SP-NLC3 formulations. Figure 3 also showed that within the first 3 months of the storage, no remarkable changes in the size of particles was observed compared to the size of nanoparticles immediately after the production. The figure also showed that, although the size of nanoparticles after 6 months at 4 and $25^{\circ} \mathrm{C}$ was increased compared to the samples stored for 3 months, this increase in the size was significantly smaller than the increase in the size of nanoparticles at $40^{\circ} \mathrm{C}$ after 6 months. This data indicated that the nanoparticles are more stable at 4 or $25^{\circ} \mathrm{C}$ compared to the samples stored at $40^{\circ} \mathrm{C}$. This can be supported by zeta potential data reported in Fig. 4. This figure showed that in the case of SLN formulations when the particles stored at different temperatures $\left(4,25\right.$ and $\left.40^{\circ} \mathrm{C}\right)$ a significant reduction $(P<0.05)$ in zeta potential was only observed for the samples stored at $40^{\circ} \mathrm{C}$ for 3 and 6 months which could be due to the aggregation of particles at $40^{\circ} \mathrm{C}$. It seems that these nanoparticles are more stable at lower temperatures. Similar results were obtained for NLC3 formulation.

These results are reflection of long-term stability of the nanoparticles at 4 and $25^{\circ} \mathrm{C}$. This observation was confirmed by the assessment of the particle size homogeneity and absence of any phase separation (22). Jenning et al. had used the wide-angle X-ray diffraction to study the polymorphic transitions of SLN made from stearic acid in oil/water cream (23). They found that after 6 months, SLNs still remained in the nanoparticulate state. The good stability might derive

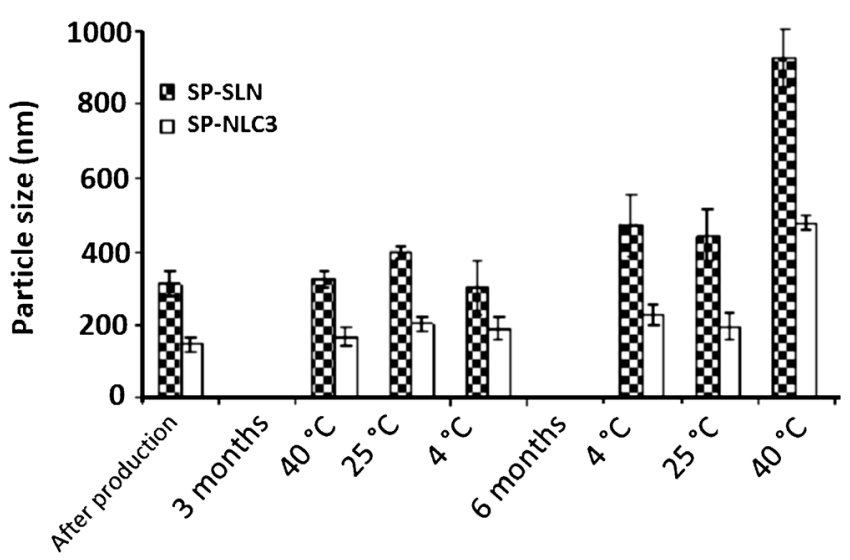

Fig. 3. Particle size of SP-SLN and SP-NLC3 formulations after production, 3 and 6 months of storage at 4,25 and $40^{\circ} \mathrm{C}$

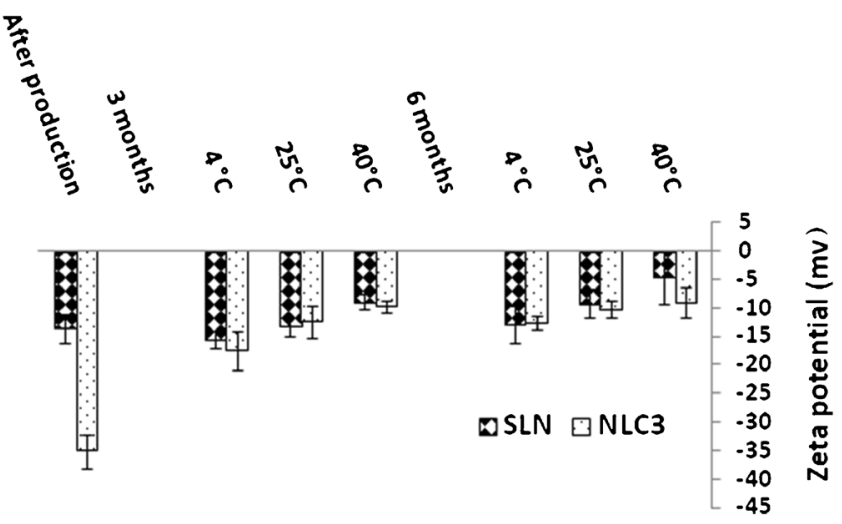

Fig. 4. Zeta potential of different formulations at different storage conditions

from the slow transition of lipid in SLN and NLC and the steric effect of surfactant (24).

Dissolution profile of freeze-dried SP-SLN, SP-NLC3 and raw drug were presented in Fig. 5. The accelerated dissolution for SP-SLN and SP-NLC3 could be mainly ascribed to the reduced particle size providing a greater surface area compared to the particle size of raw drug. The SLN and NLC powder showed burst release in the early stage of dissolution compared to raw SP. This could be due to the diffusion of un-encapsulated drug from the particles surface followed by the diffusion from the core. The homogenously distributed oleic acid in nanoparticles may have been another explanation for steady release followed by burst release of NLC formulations. Hu et al. showed that the release rate at the initial stage increased with the increasing oleic acid content in nanoparticles and this phenomenon is dependent on homogeneity of liquid lipid in solid lipid during the NLC preparation (17).

Obviously, at the initial stage, the drug release was slower in SLN compared to NLC and after that, the release profiles of SLN and NLC were almost parallel and their slopes tended to be similar. The faster drug release from NLC compared to SLN could be due to the presence of liquid lipid in the structure of NLC (25).

In conclusion, both NLC and SLN present a solid lipid matrix, but NLCs are prepared with a mixture of solid lipid and liquid lipid (oil) which increased the drug loading and stability compared to SLN formulations. This also could be due to the creation of a less ordered lipid matrix, by mixing solid lipid and liquid lipid. The major advantage of SLN is the

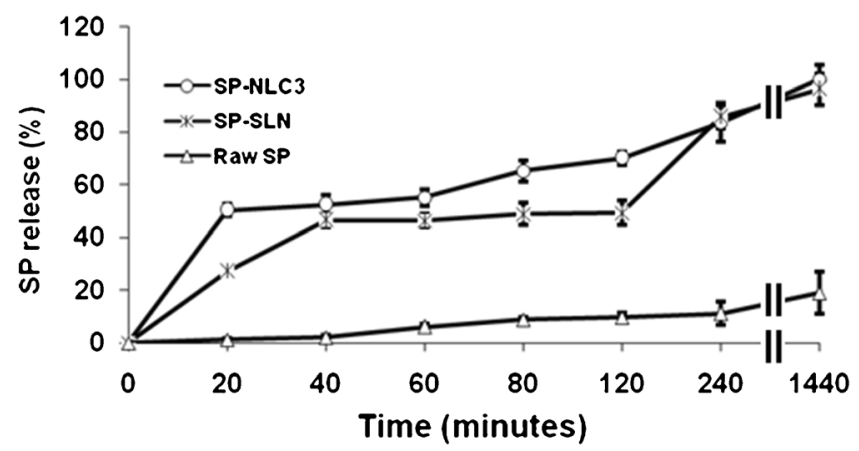

Fig. 5. Comparison of dissolution profiles of freeze-dried SP-SLN and SP-NLC3 compared to raw SP 
possibility of production on large industrial scale. However, depending on the drug some potential problems can occur, such as drug leakage during storage and insufficient total drug load. Despite these statements, controversial results have been found in the literature about the differences between SLN and NLC (26). Comparing all nanoparticle formulations indicated that in the present study, the SP-NLCs are better than SP-SLNs in terms of dissolution rates, entrapment efficiency and physical stability.

\section{CONCLUSIONS}

It can be concluded that with increasing the proportion of oleic acid in NLC, the average size and zeta potential of nanoparticles decreased significantly. Similarly, the EE increased dramatically due to the presence of interstitial spaces to accommodate the drug particles in the systems. The stability results showed that the particle size remained smaller in NLC compared to that of SLN formulations even after 6 months. The in vitro dissolution study showed about a 5.1and 7.2-fold increase in the release of the drug in $2 \mathrm{~h}$ compared to the raw drug. The effect of stabiliser on preventing aggregation of nanoparticles, both in the SLN and NLC in terms of achieving even longer-term stability can be assessed in the future.

\section{ACKNOWLEDGMENTS}

This work was partially supported by the Mazandaran University of Medical Sciences. The authors would like to thank Mrs. Choupani, Mrs. Rezanejad, Mr. Eslami and Mr. Farmoudeh for their assistance.

Open Access This article is distributed under the terms of the Creative Commons Attribution 4.0 International License (http://creativecommons.org/licenses/by/4.0/), which permits unrestricted use, distribution, and reproduction in any medium, provided you give appropriate credit to the original author(s) and the source, provide a link to the Creative Commons license, and indicate if changes were made.

\section{REFERENCES}

1. Kocbek P, Baumgartner S, Kristl J. Preparation and evaluation of nanosuspensions for enhancing the dissolution of poorly soluble drugs. Int J Pharm. 2006;312:179-86.

2. Dong Y, Ng WK, Shen S, Kim S, Tan RBH. Preparation and characterization of spironolactone nanoparticles by antisolvent precipitation. Int J Pharm. 2009;375:84-8.

3. Dong $\mathrm{Y}, \mathrm{Ng} \mathrm{WK}$, Shen $\mathrm{S}$, Kim S, Tan RBH. Controlled antisolvent precipitation of spironolactone nanoparticles by impingement mixing. Int J Pharm. 2011;410:175-9.

4. Dong $\mathrm{Y}, \mathrm{Ng} \mathrm{WK}, \mathrm{Hu}$ J, Shen $\mathrm{S}$, Tan RBH. A continuous and highly effective static mixing process for antisolvent precipitation of nanoparticles of poorly water-soluble drugs. Int J Pharm. 2010;386:256-61.

5. Laouini A, Jaafar-Maalej C, Sfar S, Charcosset C, Fessi H. Liposome preparation using a hollow fiber membrane contactor-application to spironolactone encapsulation. Int J Pharm. 2011;415:53-61.

6. Bourezg Z, Bourgeois S, Pressenda S, Shehada T, Fessi H. Redispersible lipid nanoparticles of spironolactone obtained by three drying methods. Colloids Surf Physicochem Eng Aspects. 2012;413:191-9.
7. Ohshima H, Miyagishima A, Kurita T, Makino Y, Iwao Y, Sonobe T, et al. Freeze-dried nifedipine-lipid nanoparticles with long-term nano-dispersion stability after reconstitution. Int $\mathrm{J}$ Pharm. 2009;377:180-4.

8. Kelidari HR, Saeedi M, Akbari J, Morteza-Semnani K, Gill P, Valizadeh $\mathrm{H}$, et al. Formulation optimization and in vitro skin penetration of spironolactone loaded solid lipid nanoparticles. Colloids Surf B: Biointerfaces. 2015;128:473-9.

9. Patil-Gadhe A, Pokharkar V. Montelukast-loaded nanostructured lipid carriers: part I. Oral bioavailability improvement. Eur J Pharm Biopharm. 2014;88:160-8.

10. Aditya NP, Macedo AS, Doktorovova S, Souto EB, Kim S, Chang P-S, et al. Development and evaluation of lipid nanocarriers for quercetin delivery: a comparative study of solid lipid nanoparticles (SLN), nanostructured lipid carriers (NLC), and lipid nanoemulsions (LNE). LWT Food Sci Technol. 2014;59:115-21.

11. Kumar S, Randhawa JK. High melting lipid based approach for drug delivery: solid lipid nanoparticles. Mater Sci. 2013;33: 1842-52.

12. Pardeike J, Hommoss A, Müller RH. Lipid nanoparticles (SLN, NLC) in cosmetic and pharmaceutical dermal products. Int $\mathbf{J}$ Pharm. 2009;366:170-84.

13. Müller R, Radtke M, Wissing S. Solid lipid nanoparticles (SLN) and nanostructured lipid carriers (NLC) in cosmetic and dermatological preparations. Adv Drug Deliv Rev. 2002;54:131-55.

14. Shah RM, Malherbe F, Eldridge D, Palombo EA, Harding IH. Physicochemical characterization of solid lipid nanoparticles (SLNs) prepared by a novel microemulsion technique. Colloids Surf B: Biointerfaces. 2014;428:286-94.

15. Puglia C, Blasi P, Rizza L, Schoubben A, Bonina F, Rossi C, et al. Lipid nanoparticles for prolonged topical delivery: an in vitro and in vivo investigation. Int J Pharm. 2008;357:295-304.

16. Gonzalez-Mira E, Egea MA, Garcia ML, Souto EB. Design and ocular tolerance of flurbiprofen loaded ultrasound-engineered NLC. Colloids Surf B: Biointerfaces. 2010;81:412-21.

17. Hu F-Q, Jiang S-P, Du Y-Z, Yuan H, Ye Y-Q, Zeng S. Preparation and characterization of stearic acid nanostructured lipid carriers by solvent diffusion method in an aqueous system. Colloids Surf B: Biointerfaces. 2005;45:167-73.

18. Yuan H, Wang L-L, Du Y-Z, You J, Hu F-Q, Zeng S. Preparation and characteristics of nanostructured lipid carriers for control-releasing progesterone by melt-emulsification. Colloids Surf B: Biointerfaces. 2007;60:174-9.

19. Souza LG, Silva EJ, Martins ALL, Mota MF, Braga RC, Lima EM, et al. Development of topotecan loaded lipid nanoparticles for chemical stabilization and prolonged release. Eur J Pharm Biopharm. 2011;79:189-96.

20. Marquele-Oliveira F, Santana DC, Taveira SF, Vermeulen DM, de Oliveira AR, da Silva RS, et al. Development of nitrosyl ruthenium complex-loaded lipid carriers for topical administration: improvement in skin stability and in nitric oxide release by visible light irradiation. J Pharm Biomed Anal. 2010;53:843-51.

21. Uprit S, Kumar Sahu R, Roy A, Pare A. Preparation and characterization of minoxidil loaded nanostructured lipid carrier gel for effective treatment of alopecia. Saudi Pharm J. 2013;21:379-85.

22. Ali H, Shirode AB, Sylvester PW, Nazzal S. Preparation and in vitro antiproliferative effect of tocotrienol loaded lipid nanoparticles. Colloids Surf Physicochem Eng Aspects. 2010;353:43-51.

23. Jenning V, Thünemann AF, Gohla SH. Characterisation of a novel solid lipid nanoparticle carrier system based on binary mixtures of liquid and solid lipids. Int J Pharm. 2000;199:167-77.

24. Venkateswarlu V, Manjunath K. Preparation, characterization and in vitro release kinetics of clozapine solid lipid nanoparticles. J Control Release. 2004;95:627-38.

25. Souto EB, Wissing SA, Barbosa CM, Müller RH. Development of a controlled release formulation based on SLN and NLC for topical clotrimazole delivery. Int J Pharm. 2004;278:71-7.

26. Andrade LM, de Fátima RC, Maione-Silva L, Anjos JL, Alonso A, Serpa RC, et al. Impact of lipid dynamic behavior on physical stability, in vitro release and skin permeation of genistein-loaded lipid nanoparticles. Eur J Pharm Biopharm. 2014;88:40-7. 